ИЗВЕСТИЯ АКАДЕМИИ НАУК ЭСТОНСКОИ ССР. ТОМ 26 ФИЗНКА * МАТЕМАТИКА, 1977, № 4

\title{
О МИНИМАЛЬНОЙ ЧАСТИЧНОЙ РЕАЛИЗАЦИИ СИСТЕМЫ В КАНОНИЧЕСКОЙ ФОРМЕ
}

\section{1. Введение}

Рассмотрим линейную динамическую систему

$$
\begin{aligned}
x(t+1) & =A x(t)+B u(t), \\
y(t) & =C x(t),
\end{aligned}
$$

где $x, u$ и $y$ - векторы состояния, входа и выхода размерности $n, m$ и $p$ соответственно, а $A, B$ и $C-$ матрицы подходящей размерности. Внешнее поведение системы (1) характеризуется бесконечной последовательностью матриц $M(k)$ размерности $p \times m$, определяемых формулой

$$
M(k)=C A^{k-1} B, \quad k=1,2, \ldots
$$

и называемых марковскими параметрами системы (1) [ $\left.{ }^{1}\right]$. Задача реализации заключается в определении тройки матриц $(A, B, C)$ описания (1) по заданным марковским параметрам (2). Эта проблема хорошо изучена, разработано много алгоритмов реализации $\left[{ }^{2-4}\right]$.

В практических задачах задается, как правило, конечное число марковских параметров $M(1), \ldots, M(N)$. Встает задача частичной реализации, требующая определения тройки $(A, B, C)$ такой, чтобы

$$
M(k)=C A^{k-1} B, \quad k=1, \ldots, N .
$$

Сложность системы (1) определяется, во-первых, ее размерностью $n$ и, во-вторых, количеством неизвестных параметров в тройке $(A, B, C)$. Поэтому целесообразно искать минимальную частичную реализацию ( $n$ минимальна) в некоторой кканонической форме (часть элементов матриц $A, B, C$ фиксирована). Проблема 'минимальной частичной реализации решена в $\left[{ }^{1,5}\right]$. Проблема частичной реализации в канонической форме исследована в $\left[{ }^{6-8}\right]$. Затруднения возникают с гарантированием минимальности и эквивалентности получаемых частичных реализаций $[5,8]$.

В настоящей работе рассматриваются возможности получения мимимальной частичной реализации в канонической форме Луэнбергера. Задача частичной реализации имеет много общего с задачей реализации. Процедуру Луэнбергера $\left[{ }^{4}\right]$ можно с некоторыми изменениями перенести и на частичную реализацию. Однако задача частичной реализзации имеет и свои опецифические черты. Процедура Луэнбергера 
гарантирует управляемость и наблюдаемость получаемой реализации. Оказывается, что полностью управляемая и наблюдаемая частичная реализация еще не обязательно минимальна. В данной работе выводятся дополнительные ограничения, выполнение которых гарантирует минимальность частичной реализации. Полученные результаты иллюстрируются примером из $\left[{ }^{5}\right]$. Показывается, что алгоритм частичной реализации, приведенный в $\left[{ }^{6}\right]$, не обеспечивает минимальность реализации.

\section{2. Частичная реализация в канонической форме}

Составим ганкелеву матрицу в виде

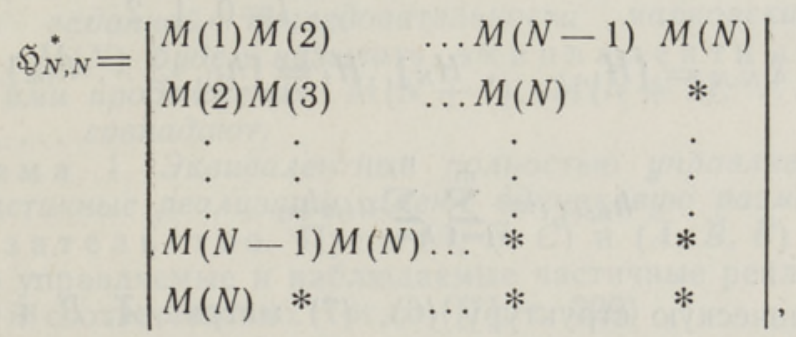

где * обозначает неизвестные матрицы размерности $p \times m$ в последовательности $\{M(N+1), M(N+2), \ldots\}$. В силу соотношений (2) ганкелева матрица $\mathscr{\bigotimes}_{\alpha, \beta}$ может быть представлена произведением

$$
\mathfrak{S}_{\alpha, \beta}=\mathfrak{D}_{\alpha} \mathscr{夭}_{\beta}=\left[\begin{array}{c}
C \\
C A \\
\cdot \\
\dot{C A^{\alpha-1}}
\end{array}\right]\left[B, A B, \ldots, A^{\beta-1} B\right],
$$

где $\mathfrak{D}_{\alpha}$ и $\mathfrak{C}_{\beta}-$ матрицы наблюдаемости и управляемости соответственно.

Если $\beta \geqslant n$, то для управляемой системы ранг $\mathfrak{夭}_{\beta}=n$. Д. Луэнбергером разработана процедура составления неособенной матрицы $T$ из $n$ линейно независимых столбцов матрицы $\mathfrak{夭}_{\beta}$, приводящей пару $(A, B)$ преобразованием

$$
\widetilde{A}=T^{-1} A T, \quad \widetilde{B}=T^{-1} B, \quad \widetilde{C}=C T
$$

в каноническую форму

$$
\begin{aligned}
& \tilde{A}=\left[A_{i j}\right], \quad i, j=1, \ldots, m, \\
& \underset{\left(n_{i} \times n_{i}\right)}{A_{i i}}=\left[\begin{array}{c:c}
0^{T} & a_{i i 1} \\
\hdashline I_{n t-1} & a_{i i n t}
\end{array}\right] \\
& \underset{\left(n_{i} \times n_{j}\right)}{A_{i j}}=\left[\begin{array}{cc} 
& \vdots a_{i j 1} \\
0 & \vdots \\
\vdots & a_{i j n j}
\end{array}\right], \quad i \neq j . \\
& \widetilde{B}=\left[e_{1}, e_{n_{1}+1}, \ldots, e_{n_{1}+\ldots+n_{m-1}+1}\right] \text {, }
\end{aligned}
$$

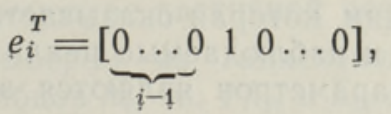


где $n_{i}$ - структурные индексы системы [ $\left.{ }^{4}\right]$. Элементы $a_{i j k}$ удовлетворяют соотношениям

$$
A^{n}, b_{i}=\sum_{j=1}^{m} \sum_{k=1}^{n_{j}} a_{i j k} A^{k-1} b_{j}, \quad i=1, \ldots, m,
$$

где $b_{i}-i$-й столбец матрицы $B$.

Если теперь ранг $\mathfrak{D}_{\alpha}=n$, то из уравнений (5) и (8) получаем рекуррентные соотношения

$$
\begin{aligned}
C A^{n_{t}+l} b_{i}=\sum_{j=1}^{m} \sum_{k=1}^{n_{j}} a_{i j k} C A^{k-1+l} b_{j}, & i=1, \ldots, m, \\
& l=0,1,2, \ldots
\end{aligned}
$$

или, обозначая $\mathfrak{S}_{N, N}=\left[H_{1}, \ldots, H_{N}\right], H_{i}=\left[h_{i 1}, \ldots, h_{i m}\right],-$ соотношения

$$
h_{n t+1, i}=\sum_{j=1}^{m} \sum_{k=1}^{n_{j}} a_{i j k} h_{k j} .
$$

Учитывая каноническую структуру (6), (7) матриц $\widetilde{A}, \widetilde{B}$ и соотношение $\mathfrak{S}_{\alpha, \beta}=\tilde{\mathfrak{D}}_{\alpha} \tilde{\mathfrak{E}}_{\beta}$, где $\tilde{\mathfrak{D}}_{\alpha}=\mathfrak{D}_{\alpha} T, \tilde{\mathfrak{E}}_{\beta}=T^{-1} \mathfrak{夭}_{\beta}$, для матрицы $\boldsymbol{C}=$ $=\left[c_{1}, \ldots, c_{m}\right], c_{i}=\left[c_{i 1}, \ldots, c_{\text {int }}\right]$ получаем

$$
c_{i k}=m_{i}(k) \text {, }
$$

где $m_{i}(k)-i$-й столбец матрицы $M(k)$. Значит, для полностью наблюдаемой системы можно найти реализацию в канонической форме Луэнбергера (6), (7) прямо из ганкелевой матрицы \$ $\alpha, \beta$.

Выделим в процедуре реализации два основных этапа:

1) соответственно алгоритму Луэнбергера $\left[{ }^{4}\right]$ определяются линейно независимые столбцы ганкелевой матрицы $\mathfrak{S}_{\alpha, \beta}$; при этом выясняются стриктурные индексы $n_{i}, i=1, \ldots, m$;

2) определяются неизвестные элементы матриц $\widetilde{A}$ и $\widetilde{C}$ из уравнений (9) и (10).

При частичной реализации возникает необходимость в модификации алгоритма Луэнбергера (ввиду того, что столбцы матрицы определены неполностью). Осуществляется это следующим образом.

1. Некоторый столбец $h_{h j}$ включается в кандидаты в базис, если: а) столбцы $h_{1 j}, \ldots, h_{k-1 . j}$ уже вошли в его состав и б) все выбранные базисные столбцы определены до длины $(N-k+1) p$ или больше.

2. Кандидат $h_{k j}$ включается в базис, если он не зависит линейно от кусков уже выбранных базисных столбцов длиною $(N-k+1) p$.

Для выполнения требования 16 некоторые базисные столбщы должны быть дополнены элементами из продолжений марковских параметров $M(N+1), M(N+2) \ldots$, вычисленных по формуле (9).

\section{3. Минимальность и эквивалентность}

Из теории реализации известно $\left[{ }^{2}\right]$, что в марковских параметрах (2) отражается лишь управляемая и наблюдаемая часть системы. Поэтому особое внимание уделяется нахождению полностью управляемой и наблюдаемой реализации, которая оказывается и минимальной. А две полностью управляемые и наблюдаемые реализации данной последовательности марковоких параметров яввляются эквивалентными, 
При частичной реализации эти утверждения не выполняются. Варьированием продолжений марковских параметров $M(N+1), M(N+2) \ldots$ можно добиться множества неэквивалентных полностью управляемых и наблюдаемых частичных реализаций заданных марковских параметров $M(1), \ldots, M(N)$. Размерность такой реализации может приобрести при этом сколь угодно большие значения. Поэтому вопросы минимальности и эквивалентности имеют особое значение для задачи частичной реализации.

О пр ед ел ен и е 1 . Частичную реализацию $(A, B, C)$ будем называть м и н и а льно й, если размерность $n$ есть минимальное число такое, что соотношения (3) выполняются.

Определени е 2 . Две частичные реализации $(A, B, C) u$ $(\bar{A}, \bar{B}, \bar{C})$ заданной последовательности марковских параметров $M(1), \ldots, M(N)$ будем называть эк в и в л е н н ы м и, если генерируемые ими продолжения $M(N+1), M(N+2), \ldots$ и $\bar{M}(N+1)$, $\bar{M}(N+2), \ldots$ совпадают.

т еорем а 1. Эквивалентные полностью управляемые и наблюдаемые частичные реализации имеют одинаковую размерность.

Д ок а з а т е л ь с т в о. Пусть $(A, B, C)$ и $(\bar{A}, \bar{B}, \bar{C})$ эквивалентные полностью управляемые и наблюдаемые частичные реализации размерности $n$ и $\bar{n}$ соответственно. Тогда ([2], с. 329$)$

$$
\begin{gathered}
\varrho \mathfrak{S}_{n, n}=\varrho \mathscr{S}_{n+\alpha, n+\beta}=n, \\
\varrho \overline{\mathscr{S}}_{\bar{n}, \bar{n}}=\mathrm{Q} \overline{\mathfrak{S}}_{n+\alpha, n+\beta}=\bar{n}, \\
\alpha, \beta=1,2, \ldots,
\end{gathered}
$$

где $\mathfrak{S}_{n, n}$ и $\overline{\mathfrak{g}}_{\bar{n}, \bar{n}}$ ганкелевы матрицы размерности $p n \times m n$ и $p \bar{n} \times m \bar{n}$, составленные из последовательностей марковских параметров $M(1), \ldots$, $M(N), M(N+1), \ldots$ и $\bar{M}(1), \ldots, \bar{M}(N), \bar{M}(N+1), \ldots$ соответственно, a @\$ обозначает ранг матрицы $\$$. Предположим, что $n<\bar{n}$. Тогда

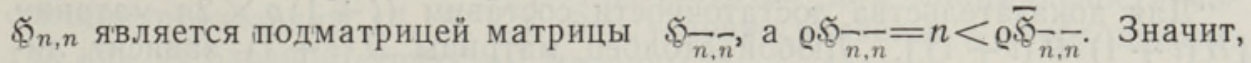
$\mathfrak{S}_{n, n}^{-} \neq \overline{\mathfrak{S}}_{\bar{n}, \bar{n}}, \quad$ т. е. частичные реализации $(A, B, C)$ и $(\bar{A}, \bar{B}, \bar{C})$ неәквивалентны.

Следствие 1. Полностью управляемая и наблюдаемая частичная реализация является минимальной, если она эквивалентна с минимальной частичной реализацией.

Одинаковая размерность является необходимым условием эквивалентности частичных реализаций. Для выведения достаточных условий әквивалентности используем лемму из [ $\left.{ }^{9}\right]$.

Л ем м а. Пусть матрицы $X, Y$ и $Z$ такие, что

$$
\mathrm{e} X=\mathrm{\varrho}[X: Y]=\mathrm{\varrho}\left[\frac{X}{Z}\right]=r \text {. }
$$

Тогда существует единственная матрица W такая, что

$$
\mathrm{e}\left[\begin{array}{c:c}
X & Y \\
\hdashline Z & W
\end{array}\right]=r .
$$

Вернемся теперь к процедуре частичной реализации в канонической форме Луэнбергера. Выберем из ганкелевой матрицы $\mathfrak{\wp}_{N, N}^{*}$ два, комплекта базисных столбцов $h_{1}, \ldots, h_{n}$ и $\bar{h}_{1}, \ldots, \bar{h}_{n}$, определим соот- 
ветствующие частичные реализации $(A, B, C)$ и $(\bar{A}, \bar{B}, \bar{C})$ и найдем продолжения марковских параметров $M(N+1), \ldots$ и $\bar{M}(N+1), \ldots$. Пусть $v_{i}(l)$ и $w_{i}(l), i=1, \ldots, n$, векторы, составленные из элементов матриц $M(k)$ и $\bar{M}(k), k>N$, соответственно так, что они дополняют столбцы $h_{i}$ в матрице $\mathfrak{\bigotimes}_{N, N}^{*}$ до длины $l p$. Аналогично, пусть векторы $\bar{v}_{j}(l)$ и $\bar{w}_{j}(l)$ дополняют векторы $\bar{h}_{j}, j=1, \ldots, n$, до длины $l p$. Отметим, что длина вектора $h_{i}$ зависит от порядкового номера соответствующего ему блок-столбца $H_{s}$ матрицы $\mathfrak{\mathscr { \vartheta }}_{N, N}^{*}$ и равняется $(N-s+1) p$. Тогда длина векторов $v_{i}(l)$ и $w_{i}(l)$ равняется $(l+s-N-1) p$. Если $k \leqslant N-l+1$, то векторы $v_{i}(l)$ и $w_{i}(l)$ пустые. Составляем матрицы $H(l)$ и $\bar{H}(l)$ размерности $l p \times n$ из базисных столбцов $h_{i}$ и $\bar{h}_{j}$, дополненных векторами $v_{i}(l)$ и $\bar{w}_{j}(l)$ соответственно:

$$
\begin{aligned}
& H(l)=\left[\begin{array}{l:l}
I_{l p} & 0
\end{array}\right]\left[\begin{array}{c:c:c}
h_{1} & h_{n} \\
\hdashline v_{1}(N) & \ldots & v_{n}(N)
\end{array}\right],
\end{aligned}
$$

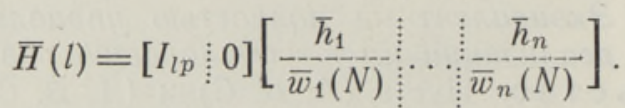

Т еорем а 2. Две частичные реализации $(A, B, C) u(\bar{A}, \bar{B}, \bar{C})$ заданной последовательности марковских параметров $M(1), \ldots, M(N)$ являются эквивалентными тогда и только тогда, когда генерируемье ими дополнения $\bar{v}_{j}(l+1) \quad u \bar{w}_{j}(l+1)$ совпадают

$$
\bar{v}_{j}(l+1)=\bar{w}_{j}(l+1), \quad j=1, \ldots, n,
$$

причем $l$ число такое, что

$$
\mathrm{\varrho} H(l+1)=\mathrm{\varrho} H(l) .
$$

Доказательство. Необходимость выполнения равенства (11) очевидна. В противном случае при некотором $k>N$ получим $M(k) \neq$ $\neq \bar{M}(k)$.

Для доказательства достаточности составим $(l+1) p \times 2 n$ матрицу $[H(l+1) \vdots \bar{H}(l+1)]$. Любой столбец матрицы $\bar{H}(l+1)$ зависит линейно от столбцов матрицы $H(l+1)$ в силу предположения (11). Учитывая еще равенство (12), получим

$$
\mathrm{\varrho}[H(l+1) \vdots \bar{H}(l+1)]=\mathrm{\varrho} H(l+1)=\mathrm{\varrho} H(l)=\mathrm{\varrho}[H(l) \vdots \bar{H}(l)] .
$$

Выберем из матрицы $\mathfrak{\mathcal { g }}_{N, N}^{*}$, дополненной элементами векторов $\bar{v}_{j}(l+1)$, $j=1, \ldots, n$, некоторый столбец $h$ длиною $l p$. Пусть векторы $v(l+1)$ и $w(l+1)$ из продолжений $M(k)$ и $\bar{M}(k), k>N$, соответственно дополняют столбец $h$ до длины $(l+1) p$. Из процедуры реализации следует

$$
\mathrm{\varrho}[H(l) \vdots h]=\mathrm{Q}[\bar{H}(l) \vdots h]=\mathrm{Q}[H(l) \vdots \bar{H}(l)],
$$

$\varrho\left[\begin{array}{c:c}H(l+1) & h \\ \hdashline w(l+1)\end{array}\right]=\varrho H(l+1) \quad$ и $\quad \varrho\left[\bar{H}(l+1): \frac{h}{v(l+1)}\right]=\mathrm{Q} \bar{H}(l+1)$.

Учитывая еще соотношение (13), получим по лемме $v(l+1)=w(l+1)$. Поочередным дополнением матрицы $\mathfrak{S}_{N, N}^{*}$ и повторным использованием леммы можно показать, что продолжения $M(k)$ и $\bar{M}(k)$ $k=N+1, N+2, \ldots$, совпадают при выполнении предположений (11) и $(12)$, т. е. частичные реализации $(A, B, C)$ и $(\bar{A}, \bar{B}, \bar{C})$ эквивалентны. 
Пусть $v-$ минимальное число такое, что

$$
\varrho \Im_{v, N-v}=\varrho \overleftarrow{צ}_{v+1, N-v} .
$$

Следствие 2. Если все базисные столбцы $h_{1}, \ldots, h_{n}$ oпределены последовательностью заданных марковских параметров $M(1), \ldots$ $\ldots, M(N)$ до длины $p(v+1)$ или больше, то соответствующая полностью управляемая и наблюдаемая частичная реализация $(A, B, C)$ будет минимальной.

Из предположения следствия 2 следует, что $\varrho \overleftarrow{צ}_{v, N-v+1}=\varrho \mathscr{Y}_{v, N-v}$. Значит, базисные столбцы минимальной частичной реализации $(\bar{A}, \bar{B}, \bar{C})$, определенной в $\left[{ }^{7}\right]$, выбираются из матрицы $\mathfrak{\mathcal { G }}_{N, N-v}^{*}$. Так как $\mathrm{\varrho} H(v+1)=\mathrm{\varrho} H(v)$, то требование $(11)$ всегда выполняется при $l=v$, и частичная реализация $(A, B, C)$ по теореме 2 является эквивалентной с минимальной частичной реализацией $(\bar{A}, \bar{B}, \bar{C})$, а по следствию 1 минимальной.

Если некоторые базисные столбцы не определены до длины $(v+1) p$, то для достижения минимальной частичной реализации надо руководствоваться следующей теоремой.

Т ео рем а 3. Частичная реализация $(A, B, C)$ заданной последовательности марковских параметров $M(1), \ldots, M(N)$ является минимальной тогда и только тогда, когда

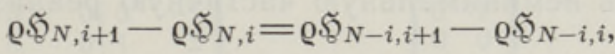

$$
\begin{aligned}
& i=1, \ldots, N-1 \text {. }
\end{aligned}
$$

Доказ а тельство. Минимальная размерность $n_{\min }$ частичной реализации последовательности $M(1), \ldots, M(N)$ определяется формулой [ $\left.{ }^{1}\right]$

$$
n_{\min }=\sum_{i=1}^{N-1}\left(\varrho \breve{G}_{N-i, i+1}-\varrho \breve{S}_{N-i, i}\right)
$$

Значит, выполнение условия (15) достаточно для минимальности частичной реализации $(A, B, C)$. Это условие является и необходимым, так как из блочной структуры матрицы $\mathfrak{g}_{N, i+1}$ следует

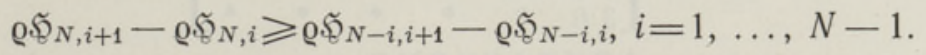

Следствие 3. Если кандидаты в базисные столбцы въыбраны в последовательности $h_{11}, h_{12}, \ldots, h_{1 m}, h_{21}, h_{22}, \ldots$, то частичная реаливация будет минимальной.

При такой последовательности выбора столбцов матрицы $\mathfrak{\xi}_{N, N}^{*}$ процедура Тетера [ ${ }^{1}$ ] гарантирует выполнение требований (15).

Следстви е 4. Eсли

$a$

$$
\begin{aligned}
& \mathrm{Q}\left[\mathscr{S}_{k, N-k} \vdots h_{i}\right]=\mathrm{Q} \breve{\mathcal{E}}_{k, N-k},
\end{aligned}
$$

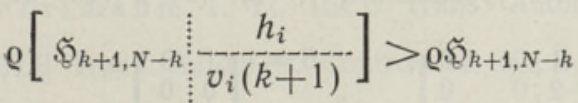

для некоторого $i \in\{1, \ldots, n\}$, то соответствующая частичная реализация $(A, B, C)$ не будет минимальной.

Для доказательспва отметим, что при удовлетворении соотношений (16) и (17) $\varrho \oiint_{N, N-k+1}-\mathrm{\varrho} \mathscr{\vartheta}_{N, N-k}>\mathfrak{g}_{k, N-k+1}-\mathrm{Q} \mathfrak{צ}_{k, N-k}$. 


\section{4. Алгоритм минимальной частичной реализации}

Учитывая полученные в п. 3 результаты, представим последовательность операций для достижения минимальной частичной реализации в канонической форме Луэнбергера.

1. Определяем индекс $v$, удовлетворяющий уравнению (14).

2. Выделяем из матрицы $\mathfrak{S}_{N, N}^{*}$ подматрицу $\mathfrak{S}_{v+1, N-v}$ размерности $(v+1) p \times(N-v) m$.

3. Выбираем из матрицы $\mathfrak{g}_{v+1, N-v}$ базисные столбцы соответственно процедуре Луэнбергера $\left[{ }^{4}\right]$. Если все базисные столбцы входят в матрицу $\$_{v+1}, N-v$, то по следствию 2 частичная реализация будет минимальной.

4. После просмотра всех кандидатов в базисные столбцы из матрицы $\S_{v+1}, N-v$ начинаем выбор базисных столбцов в последовательности $h_{N-v+1,1}, h_{N-v+1,2}, \ldots$. . По следствию 3 частичная реализация будет минимальной.

5. Определяем матрицы $A, B$ и $C$ из уравнений (6), (7), (9) и (10).

Последовательность шага 4 может быть и иной. По модифицированной процедуре Луэнбергера, представленной в п. 2, можно приступить к просмотру столбцов $h_{N-v+k, j}, j=1, \ldots, m ; k=1,2 \ldots$, раньше просмотра всех кандидатов из матрицы $\mathfrak{g}_{v+1, N-v}$. Может быть изменен и порядок перебора столбцов $h_{N-v+k, j}$. Однако в этом случае возникает опасность получить неминимальную частичную реализацию.

\section{Пример}

Рассмотрим пример Калмана [5]. Даны марковские параметры $M(1)=\left[\begin{array}{ll}1 & 1\end{array}\right]$, $M(2)=\left[\begin{array}{ll}1 & 2\end{array}\right], M(3)=\left[\begin{array}{ll}1 & 3\end{array}\right], M(4)=\left[\begin{array}{ll}2 & 4\end{array}\right], M(5)=\left[\begin{array}{ll}1 & 5\end{array}\right], M(6)=\left[\begin{array}{ll}3 & 6\end{array}\right]$, т. е. $m=2, p=1$, $N=6$.

Составляем матрицу

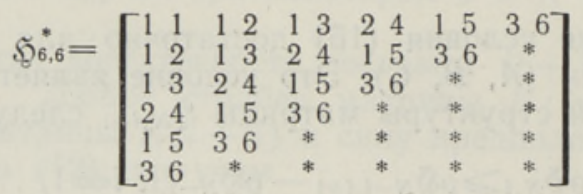

и находим индекс $v=4$.

Выделяем подматрицу $\mathfrak{S}_{v+1}, \quad N-v$

$$
\S_{5,2}=\left[\begin{array}{llll}
1 & 1 & 1 & 2 \\
1 & 2 & 1 & 3 \\
1 & 3 & 2 & 4 \\
2 & 4 & 1 & 5 \\
1 & 5 & 3 & 6
\end{array}\right]
$$

Все столбцы матрицы $\mathfrak{S}_{5,2}$ линейно независимы, а столбцы $h_{31}$ и $h_{32}$ зависят линейно от них. Значит, частичная реализация будет минимальной с размерностью $n=4$, причем $n_{1}=2, n_{2}=2$. Из уравнений $(6),(7)$, (9) и (10) находим

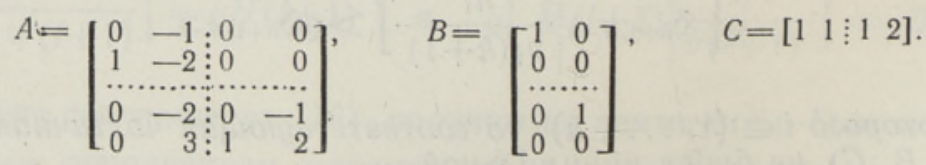

Воспользуемся теперь алпоритмом Акерманна [6] при тех же исходных данных. Для подсистемы первого входа базисными столбцами будут $h_{1}=h_{11}, \quad h_{2}=h_{21}$, 
$\bar{h}_{3}=h_{31}$, т. е. $\bar{n}_{1}=3$. Так как столбец $h_{31}$ имеет лишь $4<v+1=5$ известного элемента, следует вычислить продолжение $\bar{w}_{3}(v+1)$. Из уравнений $(6),(7),(9)$ и (10) определяем.

$$
\bar{A}_{11}=\left[\begin{array}{rrr}
0 & 0 & 2 \\
1 & 0 & 1 \\
0 & 1 & -1
\end{array}\right], \quad \bar{B}_{11}=\left[\begin{array}{l}
1 \\
0 \\
0
\end{array}\right], \quad \bar{c}_{1}^{T}=\left[\begin{array}{lll}
1 & 1 & 1
\end{array}\right]
$$

и

$$
\bar{w}_{3}(5)=\bar{c}_{1}^{T} \bar{A}_{11}^{4} \bar{b}_{11}=2 .
$$

Проверяем выполнение требований (16) и (17):

$$
\begin{gathered}
\varrho\left[\Im_{4,2}\right. \\
\left.h_{31}\right]=4, \\
\varrho\left[\begin{array}{c:c} 
& h_{31} \\
\Im_{5,2} & \vdots \ldots . . \\
\bar{w}_{3}(5)
\end{array}\right]=5 .
\end{gathered}
$$

По следствию 4 частичная реализация $(\bar{A}, \bar{B}, \bar{C})$ не может быть минимальной. Действительно, продолжая процедуру Акерманна, получаем $\bar{h}_{4}=h_{12}, \bar{h}_{5}=h_{22}$, т. е. $\bar{n}_{2}=2$, а $\bar{n}=\bar{n}_{1}+\bar{n}_{2}=5>n$. Соответствующая частичная реализация имеет вид

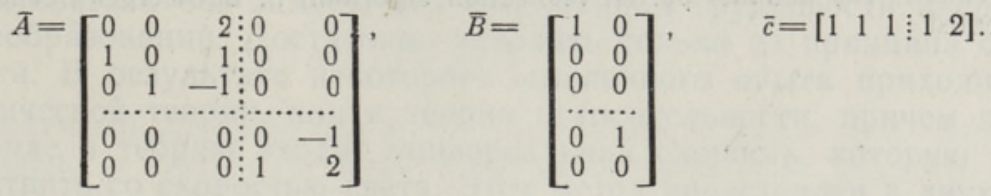

По теореме 1 частичные реализации $(A, B, C)$ и $(\bar{A}, \bar{B}, \bar{C})-$ неэквивалентны.

\section{Заключение}

Изучена процедура Луэнбергера для достижения частичной реализации в канонической форме. Выяснено, что не все разновидности модифицированной процедуры Луэнбергера дают минимальные и эквивалентные между собой частичные реализации. Выведены необходимые и достаточные условия эквивалентности и минимальности частичных реализаций. Разработан алгоритм для достижения минимальной частичной реализации в канонической форме Луэнбергера.

\section{Л И Т Е Р А Т У Р А}

1. T e the r, A. J., IEEE Trans. Automatic Control, 15, No. 4, 427 (1970).

2. Калман Р., Фалб Л., Арби б М., Очерки по математической теории систем, M., 1971 .

3. Silverma n, L. M., IEEE Trans. Automatic Control, 16, No. 6, 554 (1971).

4. L u en berger, D. E., IEEE Trans. Automatic Control, 12, No. 3, 290 (1967).

5. Kalman, R. E., In: Aspects of Network and System Theory, New York, 1971, p. 385 .

6. A ckerm a n n, J. E., IEEE Trans. Automatic Control, 17, No. 3, 381 (1972).

7. Rom a n, R., Bullock, T. E., IEEE Trans. Automatic Control, 20, No. 4, 529 (1975).

8. Ledwich, G., Fortmann, T. E., IEEE Trans. Automatic Control, 19, No. 5, 625 (1974).

9. God bole, S., IEEE Trans. Automatic Control, 17, No. 1, 173 (1972).

Институт кибернетики

Академии начк Эстонской ЭССР
Поступила в редакцию 28/X 1976 
U. NURGES

\section{SUSTEEMI MINIMAALSEST OSALISEST REALISEERINGUST KANOONILISEL KUJUL}

Vaadeldakse Luenbergeri protseduuri [3] rakendamise võimalusi osalise realisatsiooni ülesandes. On leitud osaliste realiseeringute ekvivalentsiks ja minimaalsuseks tarvilikud ja piisavad tingimused ning esitatud algoritm minimaalse osalise realiseeringu saamiseks Luenbergeri kanoonilisel kujul.

U. NURGES

ON MINIMAL PARTIAL REALIZATION IN THE CANONICAL FORM

Luenberger's procedure for solving the partial realization problem is considered. Necessary and sufficient conditions for equivalence and minimality of partial realizations are developed. The minimal partial realization algorithm in Luenberger's canonical form is presented. 